\title{
Mini Review-Qualitative Review on the Causes of Low Birth Weight
}

\section{Kana Serizawa*, Atsushi Ogihara}

Graduate school of Human sciences, Waseda University, japan

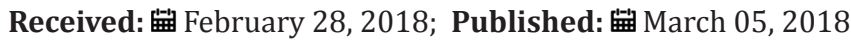

*Corresponding author: Kana Serizawa, Graduate school of Human sciences, Waseda University, Tokyo, Japan

\section{Abstract}

Object : To review the literatures on the causes of low birth weight.

Method: On the Pub Med and Ichushi (Japan Medical Abstracts Society) websites, the literatures on the causes of low birth weight published between 1975 to 2011 were searched for the

Results: From among the 469 original articles retrieved, 65 articles containing any description of the cause of low birth weight were identified, of which the 32 articles well-structured in the format of scientific writing were selected and reviewed.

Conclusion: The suspected causes of low birth weight from pre-pregnancy risks were classified into two categories:

a) Family risk factors,

b) Mother herself risk factors.

Further large-scale follow-up studies from birth would be needed at the prefectural level. .

Keywords: Low birth weight; Risk factors; Pre-pregnancy

\section{Introduction}

In Japan, the mean birth weight has been on a downward trend after peaking at $3200 \mathrm{~g}$ in 1974 , falling to $3000 \mathrm{~g}$ in 2009 . The rate of low birth weight infants has been increasing in the population of full-term infants [1]. This study aimed at sorting out the causes of low birth weight through the systematic review on the article investigating such causes.

\section{Methods}

On the Pub Med and Ichushi (Japan Medical Abstracts Society) websites, searching for the keywords listed below retrieved 469 articles (as of August 16, 2011), from among which 65 articles were selected based on title and abstract. Through a detailed review on those 65 articles, 32 articles were extracted; and another two articles on the risk factors of low birth weight were added.The search keywords: ("infant, low birth weight"[Mesh Terms] OR ("infant"[All Fields] AND "low"[All Fields] AND "birth"[All Fields] AND "weight"[All Fields]) OR "low birth weight infant"[All Fields] OR ("low"[All Fields] AND "birth weight"[All Fields]) OR "low birth weight"[All Fields]) AND ("risk factors"[Mesh Terms] OR ("risk"[All
Fields] AND "factors"[All Fields]) OR "risk factors"[All Fields]) AND pre-pregnancy[All Fields]for the Pub Med website; and the same words in Japanese for the Ichushi website.

\section{Results}

The 34 articles extracted in this review were sorted out, in terms of the causes described therein, into the two categories:

a) Pre-pregnancy risk factors,

b) Pregnancy risk factor.

Then we classified

a) Pre-pregnancy risk factors in two risks.

One is Family risk factor; the other one is mothering her risk factor.

a) Family risk factors include age $(<17$ and $>34)$, race (black), poor socio-economic condition, unmarried mother, and poor education. 
b) Mother himself risk factors include parity ( 0 or $>4$ ), low BMI, low body weight, reproductive history or obstetric history (including spontaneous abortion), and low birth weight of the mother.

\section{Discussion}

\section{Discussion About Family Factors}

Family risk factors include age ( $<17$ and $>34$ ), race (black), poor socio-economic condition, unmarried mother, and poor education. All the articles containing description about age [2-7]showed that early and old ages may be or tend to be risk factors, though some of them did not reach statistical significance. However, unlike the results of this review study, Kumar et al. [8] demonstrated that lower age might be associated with reduced risk of low birth weight. This means possible difference between races, which merits further research. The articles referring to socio-economic situation or education [3-11] showed that poor situation or poor education level may be related to the risk of low birth weight. As for marital status, only one article [12] was referring to this factor, so it is difficult to reach to conclusion. Supposedly, the higher rate of low birth weight infants may be related not to being single itself, but to its associated factors, such as economic or emotional strain.

\section{Discussion about Mother Herself Risk Factors}

A number of articles [2-18] demonstrated that low body weight or low BMI of the mother is clearly related to the risk of low birth weight. The BMI Guidelines presented by JASSO (Japan Society for the Study of Obesity) and WHO (World Health Organization) give the following definitions: underweight (under $18.5 \mathrm{~kg} / \mathrm{m} 2$ ), normal weight (18.5 to $25.0 \mathrm{~kg} / \mathrm{m} 2$ ), and overweight (over $25.0 \mathrm{~kg} / \mathrm{m} 2$ ). However, some of the articles identified in this review study have been based on other standards of underweight or overweight, and the standard weight may vary between races. These are the reasons why we need to take racial differences into consideration rather than relying solely on the BMI classification. Some articles [19] reported that low birth weight of the first infant may be associated with increased risk of low birth weight of the subsequent babies. Possibly, the mother's aging over time or rise in physical activity related to child caring may contribute to such increased risk in the subsequent births. Therefore, living conditions or availability of support from partner or other neighbors should be taken into consideration.

\section{Summary}

Many studies about potential causes of low birth weight have been published in Japan and abroad, but there have been few numbers of case-control studies at the level of every prefecture. Further large-scale studies will be needed to make it possible to use the results of this review study for improvement of pregnant women guidance, which may eventually contribute to health care guidance in less developed countries.

\section{References}

1. Kouseiroudoukyoku jinkoudoutaitoukei Gaiyo.

2. Chirshantha Abeysena, Pushpa Jayawardana, Rohini de A, Seneviratne (2010) Effect of psychosocial stress and psysical activity on low birth weight. A cohort study: J Obstet Gynaecol Res 36(2): 296-303.

3. Tsuneko Babazono (1990) A case-control study of the factors related to a higher incidence of low birth weight infants. Okayama igakkai-shi 102: 549-558.

4. Ching-pyng Kuo, Shu-Hsin Lee, Wei-ya Wu, Wen-Chun Liao, Shio-Jean Lin, et al. (2010) Lee: Birth outcomes and risk factors in adolescent pregnancies. Results of Taiwanese national survey: Pediatrics International 52: 447-452.

5. Mayumi Morita, Koichi Satou, Nobuo Sugawara, et al. (1982) Causes and countermeasures for low birth weight children. Fukushima Igaku Zassi 32: 197-204.

6. Shiyu Aizawa (2007) Maternal weight gain and neonatal outcomes: Japanese Lournal of Maternal Health 48(1): 114-121.

7. S. Ganesh Kumar, HN Harsha Kumar, S Jayaram, MS Kotian (2010) Determinants of Low Birth Weight: A case control study in a district hospital in Karnataka. Indian Journal of Pediatrics 77(1): 87-89.

8. Giurgescu C, McFarlin BL, Lomax J, Craddock C, Albrecht A (2011) Racial discrimination and the black-white gap in adverse birth outcomes: a review. J Midwifery Womens Health 56(4): 362-370.

9. Tippawan Liabsuetrakul, Southern Soil-transmitted Helminths, and maternal health working group (2011) Is international or Asian citeria-based Body Mass Index associated with maternal anaemia low birthweight, and preterm births among Thai population?-An Observational study. J heakth population NUTR 29(3): 218-228.

10. Anja Poerksen, Diana B Petitti (1991) Employment and low birth weight in BLACK MOMEN: Soc Sci Med 33(11): 1281-1286.

11. Kohta Suzuki, Junko Minami, Zentaro Yamagata (2007) Maternal negative attitudes towards pregnancy as an independent risk factor for low birthweight. J Obset Gynaecol Res 33(4): 438-444.

12. Jun Bai, WF Wong, Rajanishwar Gyaneshwar, Helen C Stewart (2000) Profile of Maternal Smorkers and Their Pregnancy Outcomes in South Western Sydney. J Obstet Gynaecol Res 26(2): 127-132.

13. Adriana Schuler cavalli, Toyoho tanaka (2000) Maternal Leisure-time Physical Activities are not Determinant Risk Factors of Low Birthweight Babies: A Cross-sectional study of 1714 Pregnant Women. Environmental Health and Preventive Medicine 5(2): 72-80.

14. Hidemi Takizaw (2006) Pregnancy and nutrition metabolism aiming for proper nutrition management during pregnancy. Acta obstetrica ET Gynecologica Japonica 58(9): 1514-1518

15. Toshie Tsuda, Toshiko Kodera, Chieko Ohya (2002) Relationship between pre-pregnancy eating behavior, nutritional status and lowweight infants. The Japan Society of Home Economics 53(1): 1009-1020.

16. Hiromi Ohmi, Kenzou Hirooka, Akira Hata, Yoshikatsu Mochizuki (2001) Recent trend of increase in proportion of low birthweight infants in Japan. International Journal of Epidemiology 30(6): 1269-1271.

17. Shiyu Aizawa ,Miho Sekimoto, John I Takayama (2005) A Trial of Epidemiological Study using the Electronic Medical Records. Maternal Weight Gain and Neonatal Outcomes. Japan journal of medical informatics 24(3): 383-390.

18. Ihunnaya O, Frederick, Michelle A, Williams, Anne E Sales, et al. (2008) Pre-pregnancy Body Mass Index, Gestaional weight gain, and other maternal characteristics in relation to infant birth weight: Maternal child health J 12(5): 557-567. 
19. Noriko Fujita, Mitsuaki Mtsui, Sopha Srey, Chin Samuth Po, Sokhan Unog, et al. (2005) Antenatal care in the capital city of Combodia: Current situation and impact on obstetric outcome.J Obset Gynaecol Res 31(2): 133-139

\section{(c) \\ This work is licensed under Creative Commons Attribution 4.0 License}

To Submit Your Article Click Here:

Submit Article

DOI: $10.32474 /$ PAPN.2018.01.000111

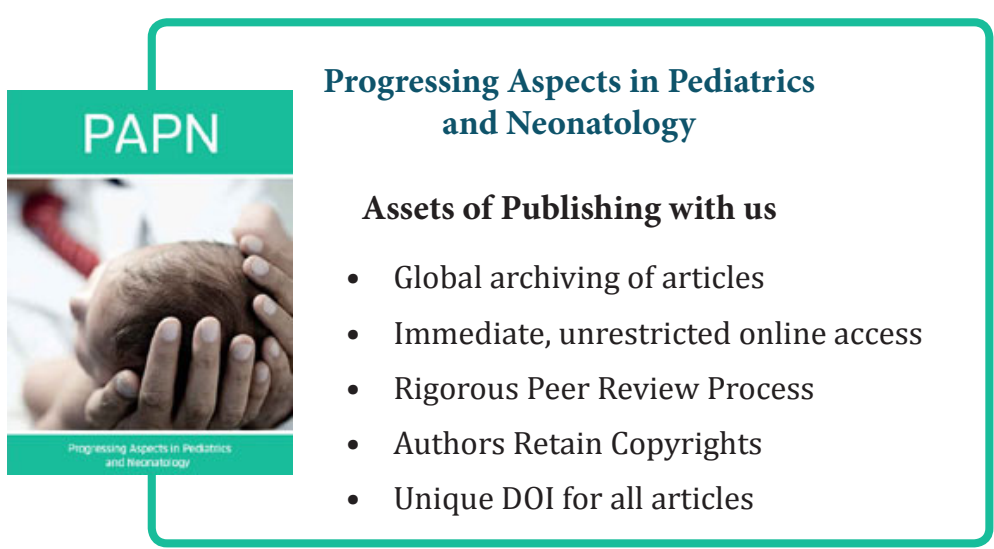

